\title{
Lack of universality in MHD turbulence, and the possible emergence of a new paradigm?
}

\author{
Annick Pouquet ${ }^{1}$, Marc-Etienne Brachet ${ }^{2}$, Ed Lee $^{3}$, \\ Pablo Mininni ${ }^{1,4}$, Duane Rosenberg ${ }^{1}$, and Vadim Uritsky ${ }^{5}$ \\ ${ }^{1}$ Geophysical Turbulence Program, National Center for Atmospheric Research, \\ PO Box 3000, Boulder CO 80304 USA \\ email: pouquet@ucar.edu and duaner@ucar.edu \\ ${ }^{2}$ École Normale Supérieure, 24 rue Lhomond, 75005 Paris, France \\ email: brachet@physique.ens.fr \\ ${ }^{3}$ Centrum voor Plasma-Astrofysica, Departement Wiskunde, Katholieke Universiteit Leuven, \\ Celestijnenlaan 200B, B-3001 Leuven, Belgium \\ email: edlee@ucar.edu \\ ${ }^{4}$ Departamento de Física, Facultad de Ciencias Exactas y Naturales, \\ Universidad de Buenos Aires, Ciudad Universitaria, 1428 Buenos Aires, Argentina \\ email: mininni@df.uba.ar \\ ${ }^{5}$ Physics and Astronomy Department, University of Calgary, Calgary, AB T2N1N4 Canada \\ email: uritsky@ucalgary.ca
}

\begin{abstract}
We review some of the recent results obtained in MHD turbulence, as encountered in many astrophysical objects. We focus attention on the lack of universality in such flows, including in the simplest case (no externally imposed magnetic field, no forcing, unit magnetic Prandtl number). Several parameters can foster such a breakdown of classical Kolmogorov scaling, such as the presence of velocity-magnetic field correlations, or of magnetic helicity and the role of the interplay between nonlinear eddies and Alfvén waves. A link with avalanche processes is also discussed. These findings have led to the conjecture of the emergence of a new paradigm for MHD turbulence, as a possibly unsettled competition between several dynamical phenomena.
\end{abstract}

Keywords. turbulence, magnetic fields, (magnetohydrodynamics:) MHD, waves, Sun: flares, (Sun:) solar wind, stars: activity, ISM: magnetic fields

\section{Introduction}

Observational data, old and new, concerning structures and statistical properties of magnetic fields, in particular in the solar photosphere and the solar wind, unambiguously speak to their ubiquity and dynamical importance. The large scales, which contain the energy, can be analyzed in the framework of the MHD limit but small scales need an approach incorporating plasma effects. One open question is: How independent are the small-scale kinetic effects and the large-scale fluid behavior? This is important in many applications of astrophysical interest, in particular in reconnection processes whereby energy is being lost to the fields (velocity and induction), resulting in particle acceleration, in heating (e.g., of the solar corona) and in dissipation of energy at a finite rate in the limit of large Reynolds number (see Biskamp \& Welter 1989 and Politano et al. 1989 for the two-dimensional (2D) case, and Mininni \& Pouquet 2009 in 3D). One of the major problems in the reconnection theory is understanding the physical mechanism of fast magnetic reconnection which seems to operate in weakly collisional space plasmas. The classical models of Sweet-Parker and Petschek approach this question in the realm of resistive MHD, but the plasmas mentioned above in fact obey a generalized Ohm's 
law in which the Hall current for fast collisionless reconnection plays a crucial role (see e.g. Wang et al. 2000 and references therein). However, numerous studies have confirmed recently that there is really no need for kinetic effects, e.g. a Hall current for reconnection (of, say, a Harris sheet, a well-known example of magnetic field reversal using a simple tanh profile) to occur provided the Reynolds number is high enough. The origin of current sheets is to be found in the nonlinear coupling of many interacting scales as happens in any turbulent flow, be it shear layers in fluids or vorticity and current sheets in MHD (Matthaeus \& Lamkin 1986, Servidio et al. 2010). Hence, there is a renewed interest in the large scale properties of conducting fluids in the MHD framework.

The interactions between widely separated scales, or nonlocal interactions (the lack of locality referring to the nonlinear coupling of modes in Fourier space), is an issue that can be addressed in the context of MHD, without reference to plasma effects at small scale. It has been thoroughly reviewed recently (Mininni 2011), and it will not be dealt with here beyond stating the important fact that, as expected on the basis of numerous studies in the mid sixties, MHD is more nonlocal than fluids; for example, reconnection (which gives rise to change in topology) involves a global structure modification because of alterations to the small scales (for Hall MHD non-locality, see Mininni et al. 2007). Furthermore, we shall not discuss either the generation of magnetic fields through dynamo action, as it is the topic of several contributions to this symposium by other authors.

We thus now review some of the results that have been obtained in recent years for MHD turbulence. Two points stand out: (i) There is now a wealth of evidence, theoretical, observational and numerical, from several groups, that universality breaks down in MHD (where universality could be defined as having one answer to one question such as the energy distribution among Fourier modes in MHD); this takes place depending on how fast the interactions of eddies and waves happen, including in the simplest case (incompressible, no Hall term, no ambipolar drift, no forcing, no uniform magnetic field); and (ii) The nature of the structures that develop in MHD flows, such as rolled-up current and vorticity sheets, as have been observed in the Solar Wind, where rotational discontinuities, flux transfer events and plasmoids are all common. We will also discuss what can be done about the limitations in power of present-day computers. Specifically, we will comment on some of the models that may allow us to explore, for example, the regimes of realistic magnetic Prandtl numbers, i.e. very small in the solar convection zone and photosphere or very large in the interstellar medium, both unreachable to this day using direct numerical simulations (DNS) of the primitive MHD equations.
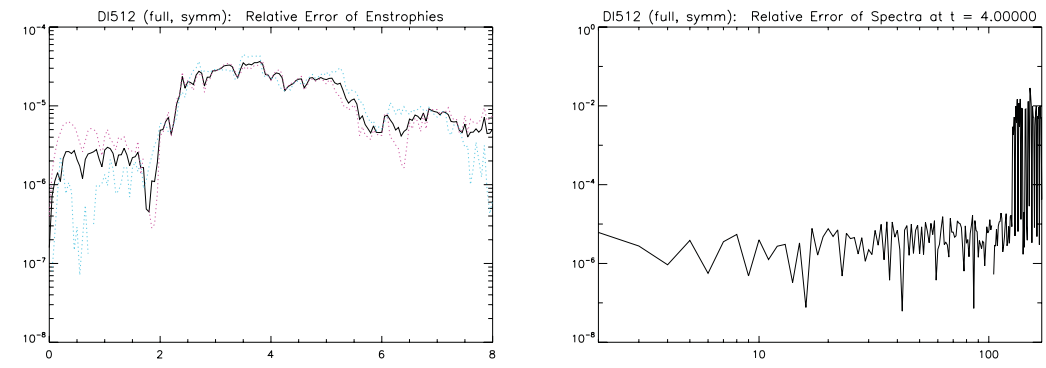

Figure 1. Left: Temporal evolution of the normalized difference of the total energy (solid line) and of its kinetic $E_{v}$ and magnetic $E_{m}$ components in a full DNS and in the symmetric code for the TG flow at the same Reynolds number. Right: Spectrum of that difference shortly after the peak of dissipation $(t=5)$. In both cases, the discrepancies are $\sim 10^{-5}$ except at the truncation. 


\section{Three energy spectra in MHD}

There has been a dispute in the literature for a long time as to whether the total energy spectrum $E_{T}(k)$ in MHD follows a Kolmogorov (1941) law $\sim k^{-5 / 3}$ (hereafter K41) as for incompressible fluids, including in the anisotropic case in the presence of a strong imposed magnetic field $\mathbf{B}_{0}$ as proposed in Sridhar \& Goldreich (1994) (see also Montgomery \& Matthaeus 1995, Ng \& Bhattacharjee 1996), or whether the interplay between Alfvén waves and nonlinear eddies slows down the nonlinear transfer to small scales, leading to a $-3 / 2$ spectrum as proposed by Iroshnikov (1965) and Kraichnan (1967) (hereafter IK), again with an anisotropic version which happens to coincide with the theory developed in the case of weak turbulence (hereafter WT), leading (phenomenologically) to $E_{2}\left(k_{\perp}, k_{\|}\right) \sim k_{\perp}^{-2} k_{\|}^{-1 / 2}$, perpendicular and parallel referring to the direction of $\mathbf{B}_{0}$, and with in the isotropic case $k_{\perp} \sim k_{\|} \sim k$. The differences in spectral indices are small and therefore hard to measure in the Solar Wind or in DNS (note that for energy spectra to converge both at small and large scales, a turbulent spectrum must have a spectral index $1<\alpha<3$, with $\left.E(k) \sim k^{-\alpha}\right)$. This includes the two-dimensional MHD case which was thoroughly investigated in the mid eighties by several teams and which corresponds, to lowest order, to reduced (anisotropic) MHD whereby the flow is reduced to the 2D plane in the presence of a strong $\mathbf{B}_{0}$. But these investigations were still somewhat inconclusive, with either IK (Biskamp \& Welter 1989, and Politano et al. 1989) or K41 scaling (see Mininni et al. 2005, in the context of a regularization model of MHD in 2D).

It is first Dmitruk et al. (2003) that showed that different spectra may occur in the case of reduced MHD; Müller \& Grappin (2005) then showed this breaking of universality in MHD turbulence, with either a K41 or IK scaling depending on the strength of the imposed field, conclusions that were confirmed by Mason et al. (2008). We recently did a study devoid of two of the main constraints of these previous works: we include no forcing, and $\mathbf{B}_{0} \equiv 0$; however, in order to obtain a sizable resolution in the inertial range in a parametric study, we impose the symmetries of a specific velocity field, the Taylor-Green vortex (or TG flow) at all times using a specialized code; such a flow has been studied extensively in the past (Brachet et al. 1983) and is akin to an experimental configuration using two counter-rotating cylinders. We couple the TG velocity in a series of three sets of numerical experiments, to three different magnetic fields, also fulfilling the same symmetries, with at $t=0$, equal kinetic and magnetic energy, identically zero magnetic helicity $H_{m}=<\mathbf{A} \cdot \mathbf{B}>$ (with $\mathbf{B}=\nabla \times \mathbf{A}$ ) and negligible velocity-magnetic field correlation $\left(H_{c}=<\mathbf{v} \cdot \mathbf{B}>\right.$ is less than $4 \%$ in normalized value for the three runs). Hence, from a statistical point of view, all three flows are identical since they have the same quadratic invariants, differing only in detailed phase factors. However, they develop markedly different spectra, one following K41, one IK and one WT (Lee et al. 2010).

The imposed symmetries do not seem to alter the dynamical evolution of the flow (see Fig. 1) and the results show as well that, in the case of the IK spectrum, indeed the relative energy $E_{v}(k)-E_{m}(k)$ follows a $k^{-2}$ law (see Fig. 2), as a simple argument can show using as a small parameter the ratio of the Alfvén to the eddy turnover timescale

$$
R_{\tau}(\ell)=\tau_{A}(\ell) / \tau_{N L}(\ell) .
$$

Fig. 2 (right) gives the anisotropic second order structure function (corresponding to the energy spectrum, with $S_{2} \sim \ell^{s}, E(k) \sim k^{-\alpha}$ and $\alpha=s+1$ ). The flow is well resolved until $\ell \sim 0.04$ (with $S_{2} \sim l^{2}$ ), and there is a clear inertial range in the perpendicular structure function (solid line) until $\ell \sim 0.7$ corresponding for this flow to a weak turbulence spectrum; the scaling of the parallel data is not as good but could possibly follow $E\left(k_{\|}\right) \sim k_{\|}^{-3 / 2}$. Note that perpendicular and parallel refer to the local uniform magnetic 

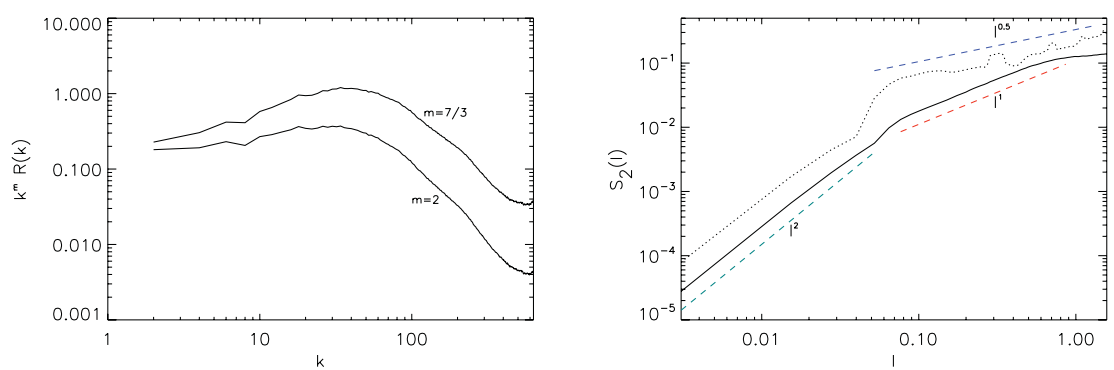

Figure 2. Left: Fourier spectrum of $E_{v}-E_{m}$ at peak of dissipation. Right: Anisotropic second-order structure function $S_{2}$ as a function of distance $\ell$; solid and dash lines correspond to perpendicular and parallel to the mean local magnetic field; at small scales, the flow is well resolved $\left(S_{2} \sim \ell^{2}\right)$, and at large scale, $S_{2}\left(\ell_{\perp}\right) \sim \ell_{\perp}$, corresponding to weak MHD turbulence.

field $\mathbf{B}_{0, \text { local }}$ in the absence of imposed uniform field as in this run; $\mathbf{B}_{0, \text { local }}$ is computed, at a cost, as an average of the fluctuating field in a sphere of radius the integral length scale and centered on the spatial location at which the structure functions are evaluated.

As a last point, we might mention that these new highly resolved numerical simulations point out to a lack of observance of what is called critical balance (Goldreich \& Sridhar 1995). Indeed, the ratio of time scales in the above equation depends on the scale $\ell$ since $\tau_{A}$ and $\tau_{N L}$ have different scaling. It was assumed in Sridhar \& Goldreich (1994) that $R_{\tau}(\ell) \sim \ell^{0}=C$ with $C=1$ (note that in Galtier et al. (2005), $C \neq 1$ leaving room for scaling laws that differ from K41). However, the numerical data shows that the constancy of $R(\ell)$ with scale is not fulfllled and in fact there is weak evidence for it following what one can expect when evaluating the eddy turn-over time in the usual manner based on the observed scaling for the energy spectrum (see Lee et al. 2010). Note that this points out to the non-uniformity of the approximation behind weak turbulence theory for which $R(\ell)$ is assumed small at all scales.

The lack of universality in MHD seems to be in agreement with several recent observations in the Solar Wind. It also corroborates the findings of different scaling laws for structure functions of varying order in solar active regions, as a function of their intensity (see e.g. Yurchyshyn et al. 2005), with more intermittency the stronger the flare; a similar tendency has been found in the turbulent component of the ultraviolet auroral emission representing multiscale magnetic disturbances in the Earth's magnetosphere (Uritsky et al. 2001), and in DNS (Müller \& Biskamp 2003). However, many questions remain open. For example, in the case of the TG study, would the results given above persist when the symmetries of the flow are not maintained? Would such results persist in time (they may differ in fact)? As the Reynolds number is increased, even when keeping the magnetic Prandtl number equal to unity? And are they the stationary solutions for such flows in the presence of forcing? These questions will require many investigations, but the fact remains that there may indeed be different energy spectra in MHD, at least given the imposed constraints of the flows studied in the several papers mentioned above.

\section{The role of helicity}

The two other quadratic invariants of the MHD equations in $3 \mathrm{D}$ in the absence of dissipation are $H_{c}$ and $H_{m}$ defined above. What role do they play? Again, old and new results shed some light on the dynamics of MHD when taking into account the helical part of the velocity and magnetic field two-point correlation functions. 


\subsection{Non-zero cross-correlations between the velocity $\mathbf{v}$ and the magnetic field $\mathbf{b}$}

The lack of universality in MHD turbulence is a well-known phenomenon when one varies the amount of correlation between the velocity and magnetic field: at high correlation, the nonlinear interactions are weakened and the spectral indices of the Elsässer variables $\mathbf{z}^{ \pm}=\mathbf{v} \pm \mathbf{b}$, with spectra $E^{ \pm} \sim k^{-m^{ \pm}}$, obey $m^{+}+m^{-}=3$. Note that it can be shown analytically in the context of weak MHD turbulence that $m^{+}+m^{-}=4$ in that case (in other words, these results should be seen in the context of IK and/or WT). This has been observed numerically for example in two dimensions using both DNS and two-point closure models of turbulence (see e.g. Pouquet $(1993,1996)$ for reviews).

Recently, Boldyrev (2006) (see also Boldyrev 2005) gave a very interesting and novel interpretation of such a scaling in MHD turbulence, finding a way to incorporate in a simple manner the effect of $H_{c}$. In the context of the exact laws that can be written in MHD at the level of third-order correlators (Politano \& Pouquet 1998), one could consider that the angle $\theta(\ell)$ between $\mathbf{v}$ and $\mathbf{b}$ plays a role in such scaling, an hypothesis which in the simplest case (of equal scaling for all variables) leads to the IK spectrum and to a dependence $\theta(\ell) \sim \ell^{1 / 4}$ (omitting anisotropy for simplicity here), as observed in several numerical simulations (Mason et al. 2006).

\subsection{Revisiting the dynamics of magnetic helicity and its role}

One can predict, on the basis of its conservation, that magnetic helicity will cascade à la Kolmogorov, that it will be to large scales because of ideal dynamics (Frisch et al. 1975), and that it will follow $H_{m}(k) \sim k^{-2}$ (thus $E_{m}(k) \sim k^{-1}$ in the maximal helical case, leading to the necessity of logarithmic corrections and to the importance of nonlocal effects, as discussed in Pouquet et al. 1976). Note that a -2 spectrum obtains irrespective of whether one takes a K41 or IK approach. The data for such an inverse cascade is ambiguous today; older studies at lower resolution or using two-point closures of turbulence are in agreement with such predictions but recent numerical results under a variety of conditions and at high resolution (up to $1536^{3}$ grid points), and thus high Reynolds numbers, are in striking disagreement (Müller 2008, Malapaka 2009, Mininni \& Pouquet 2010), with in some cases a direct cascade of $H_{m}$. The magnetic helicity cascade can be viewed as a competition between the Alfvén effect leading to a tendency toward equipartition in the small scales (a phenomenon that occurs faster the smaller the scale and faster the stronger the magnetic field in the large scales), and what one can call helicity effects dealing with an inverse transfer of magnetic helicity and thus magnetic energy to large scales (Pouquet et al. 1976). So, what is happening?

One might ask whether an invariant can present a dual (direct and inverse) cascade with dual constant flux, of one sign at large scales and the other sign at small scales, even though one direction would probably be dominant. It is the case for energy in rotating turbulence (Mininni \& Pouquet 2010), with both an inverse (quasi-2D) and a direct cascade, the latter because rotation is felt less at smaller scales and isotropy recovers beyond what is called the Ozmidov scale at which the inertial wave time and the eddy turn-over time equilibrate. Magnetic helicity presents a dual cascade as well. It certainly has dual transfer, as does $H_{c}$, to small and large scales as shown in low resolution decay computations (Pouquet \& Patterson 1978); more importantly, highly resolved MHD flows using hyperdiffusivities and small-scale forcing clearly show a dual cascade of $H_{m}$ with dual constant flux (Malapaka 2009) and with a steeper spectrum that seems to be governed by a partial Alfvénization of the flow, the inertial indices of spectra varying in such a way that $H_{v} / k E_{v} \sim k^{2} H_{m} / E_{m}$ is fulfilled, compatible with a dynamo regime as well. Observe that $k H_{m} / E_{m} \sim 1 / k$, as for Navier-Stokes (for which $H_{v} \sim E_{v} \sim k^{-5 / 3}$ ), implying a slow recovery of mirror symmetry at small scale. 
Moreover, a study of the statistical equilibria of incompressible three-dimensional MHD turbulence (Stribling \& Matthaeus 1990) indicates that the cascades of magnetic helicity and cross helicity, $H_{m}$ and $H_{c}$, are different. Whereas $H_{m}$ may undergo a complete condensation (by which is meant that it will condense on the largest scale available to the system and possibly rebound to smaller scales), the case for $H_{c}$ is not so clear: a priori, having the same physical dimension as energy, it is expected to cascade to the small scales but it may also exhibit for some values of the parameters (here, the "temperatures" associated with the three global invariants), a quasi-condensation at large scales.

How do such duality and differences in cascades and fluxes affect the dynamics in a way that we can model in simple ways? This question may be particularly relevant in the framework of recent observations in the solar wind where an inverse cascade of crosscorrelation is diagnosed for highly aligned $\mathbf{v}-\mathbf{b}$ fields (Smith et al. 2009), whereas it is direct at lower levels of correlation. These topics clearly need further investigations.

\section{Modeling of MHD flows}

There is a necessity to study fundamental phenomena in astrophysical environments but in a range of parameters too wide for DNS at high resolution. Space limitations do not permit us to deal at length with this important issue (see e.g. a recent review of the use of specific models in Pouquet et al. 2010, and for a general review of Large Eddy Simulations, Meneveau \& Katz 2000). Hybrid models are one possibility, combining kinetic and continuum physics. But it cannot be stressed enough that we are close to hitting a brick wall until new technological venues are found: miniaturisation of components is at roughly ten times the atomic scale, and the power concentration in chips is approaching that of a rocket engine. So it is best to think in terms of a three-pronged approach, combining (i) DNS - giving the most accurate description of the phenomena at hand for the primitive equations, (ii) quasi-DNS - where at a given grid size, Reynolds numbers higher, say an order of magnitude at most, than in the DNS on the same grid, can be achieved by "proper" filtering (e.g., Lagrangian method of regularization of the primitive equations, preserving the Hamiltonian structure of the equations albeit in a different norm), and (iii) sub-grid scale modeling where one introduces a wealth of turbulent transport coefficients stemming from theoretical or phenomenological studies, the alpha effect of dynamo generation possibly being the best known example in MHD in astrophysics. Other models can and should be developed and studied, and all such approaches should be combined and contrasted in a quasi-steady state, with hopefully a positive slope toward enlightenment. As the number of parameters increases with the complexity of the physics/chemistry, these developments will have to occur, the small (or large) magnetic Prandtl number regime being one such successful example. The imposing of symmetries of the TG flow is another approach, as are the studies of ideal dynamics as a precursor to dissipative phenomena (see e.g. Krstulovic et al. 2009 and references therein). Another important development to be seen in the future will be the use of more sophisticated numerical methods, not only in their scaling properties to a large number of processors but also in their adaptivity (dynamic or static, e.g. with embedded grids), keeping in mind that a high level of accuracy may prove important in the assessment of extreme phenomena such as reconnection events requiring the evaluation of steep gradients.

\section{Evidence for self-organized criticality and avalanches in MHD}

Small scale structures are dominated by current and vorticity sheets which, at high Reynolds number, undergo several types of instability at any given time (see Fig. 3). 


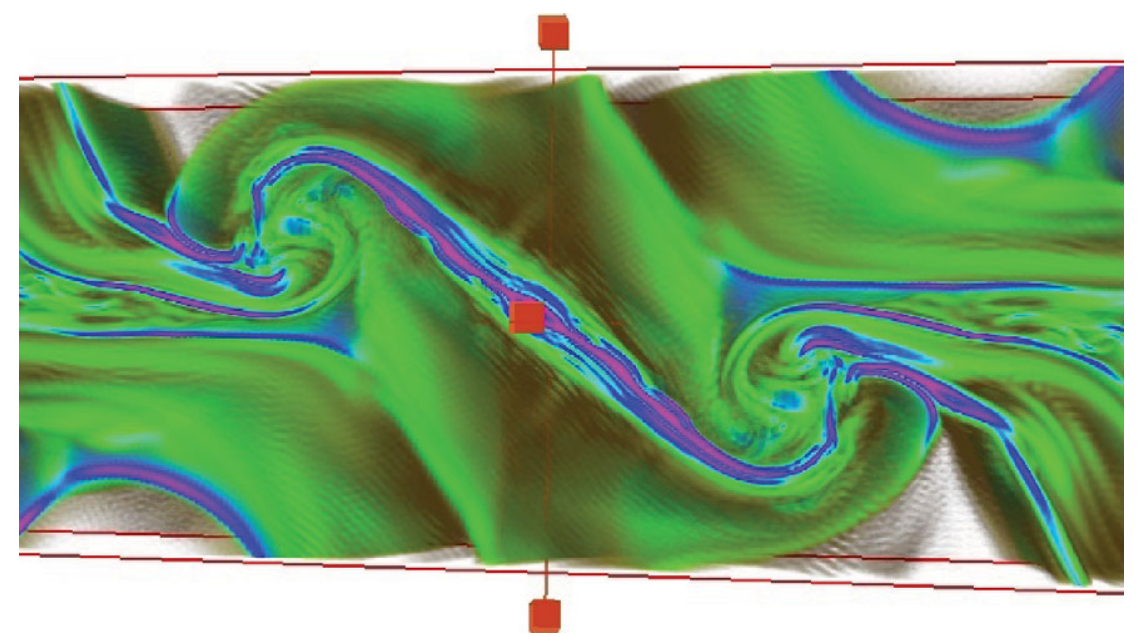

Figure 3. Zoom on current density at $\mathrm{t}=4$, TG flow with an IK spectrum, $2048^{3}$ equivalent grid resolution. Strong current sheets appear at different stages of their evolution: some are straight, some are curved, some are undergoing complex roll-up and some display secondary islands (at the center) that could be associated with avalanche-like cascades of reconnecting events.

These dissipative events may have statistics that follow self-organized criticality (SOC), as demonstrated on an avalanche model in Lu \& Hamilton (2001), and as analyzed in 3DDNS data in Uritsky et al. (2010) (note that no such avalanche behavior was discernible in the inertial range structures). The coexistence of SOC and turbulent regimes has also been recently documented for the solar corona (Uritsky et al. 2007, 2009).

We can speculate on one of the possible sources of burstiness in the dissipation and its relation with avalanches. Starting from the pioneering work of Onsager (1949), Lee (1952) and Kraichnan (1975), one can think of the dynamical evolution of a turbulent flow being due to nonlinear interactions with weak forcing and weak dissipation balancing each other. Solutions of the ideal truncated equations obtain at late times with, in the simplest instance, equipartition between all the modes, and with zero energy flux. At intermediate times and intermediate scales, one observes turbulent dynamics with non-zero flux (Cichowlas et al. 2005), the "dissipation" of large-scale energy being associated with a turbulent eddy viscosity due to the thermalized modes at small scale (see Krstulovic et al. $(2009,2010)$ for similar results for helical flows, and for 2D MHD). This dynamics has also been observed in viscous cases, e.g., in Navier-Stokes fluids and in MHD at high resolution, where the resulting flow can be decomposed, using wavelets, into a set of coherent structures with a spectrum close to Kolmogorov, and a large number of modes at small scales and in thermal equilibrium (Okamoto et al. 2007 and references therein).

Related to these results, it has been known for some time, and in different instantiations of turbulent flows, that the energy flux of a given sign on average, has in fact huge fluctuations of both signs and of amplitudes much larger than the mean (see for example Bandi et al. 2009 and references therein, and Graham et al. 2010 for studies of regions with zero flux in models of turbulence). These large fluctuations in the flux can be attributed to the balance between forcing and dissipation mentioned above, and to the two components (one thermalized and random, one turbulent and coherent) identified in turbulent flows at small scales. The interplay between the two components can result in a bursty flux transfer of energy to the small scales, as observed in particular when looking at dissipation and reconnection events (Politano et al. 1989). These bursts are 
the needed excursions that lead the system away from thermal equilibrium; thus, they may give rise to a state of criticality in order to dissipate the energy accumulated over various lapses of time through the injection mechanism. Some of these events will trigger in turn other events, by pushing around structures which then can make contact with other structures that may in turn destabilize, leading to secondary island formation and ejection of plasmoids. It will be of interest to study SOC behavior more fully in a turbulent flow, including in the presence of a Hall current, sometimes interpreted as a dissipative range although it is nonlinear and leads to a self-similar energy distribution. Will SOC behavior be identified in the Hall regime, or will it be relegated, as in MHD, to the exponentially decreasing part of the Fourier spectrum?

Note that, in our analysis of structures in several MHD configurations, it is the flow with strong $\mathbf{v}-\mathbf{b}$ correlations that exhibits SOC signatures: its critical exponents lie in the range of some well-known SOC universality classes. In general, it is possible that the lack of SOC universality in the inertial range may be caused by distinct universality classes describing SOC avalanches in the sub-inertial range. If we were to accept that SOC physics does control growth and decay of dissipative structures at these scales, then the (presumably) inverse transfer associated with avalanches at these scales would counter-stream with the direct MHD cascade. The two (small scale inverse and large scale direct) will meet roughly at the dissipation scale which may be more or less "penetrable" for the energy flux in Fourier space depending on the SOC universality class which itself may depend on the nature of the turbulence (e.g., 2D or 3D, fluid or MHD, ...).

\section{Concluding remarks}

In view of the lack of universality of energy and helicity spectra for MHD turbulence, in the presence or not of cross correlation or magnetic helicity, one may wonder what's left? Exact laws, in the simplest case under the hypotheses of isotropy, incompressibility, homogeneity, stationarity and high Reynolds number, may be one answer (see Politano \& Pouquet 1998 for MHD, Politano et al. 2003 for magnetic helicity, and Galtier 2010 for Hall MHD). In fact it has been shown, in the context of a model insuring regularization of the primitive equations (see $\S 4$ ), that the different scaling expected because of the presence of new terms in the modified " $4 / 5$ th" (Kolmogorov) exact law for this model indeed lead to a succession of energy spectral ranges that are compatible with the theory (Graham et al. 2009). So the exact laws do contain information about possibly different scaling in the specific case of MHD as well; they also show the strong link between the conservation of total energy $E_{T}$ and cross-correlation $H_{c}$ (the two are coupled), and the fact that these two equations for $E_{T}$ and $H_{c}$ may introduce a priori two time-scales (not taking into account magnetic helicity itself): this opens the door for more complex dynamics than in the incompressible fluid case. As conjectured by several authors in different ways, it is the interplay between the invariants (with given rates of transfer) and their associated time-scales (the Alfvén time, the eddy turn-over time and their combinations) that may well govern the dynamics. Moreover, anisotropy in MHD is bound to play a role, opening even more the door to a variety of dynamical equilibria.

MHD is not the only case where breaking of universality has been observed. For example, for neutral flows in the presence of rotation, one obtains a dual cascade to the small scales of kinetic energy (with spectral index $e$ ) and of (kinetic) helicity $H_{v}=\langle\mathbf{v} \cdot \omega\rangle$ (with $\omega=\nabla \times \mathbf{v}$ the voriticity), with spectral index $h$, and with $e+h=4$, at least at sufficiently high rotation at a given Reynolds number (see Mininni \& Pouquet 2010, Baerenzung et al. 2008, and Baerenzung et al. 2010). This can be understood in terms of an argument stemming from the dual considerations of (i) a cascade to small scales 
dominated by the helicity (the energy being cascaded to large scales because of the quasi bi-dimensionalization of the flow), and (ii) the transfer to small scales being mediated by inertial waves, like in MHD where Alfvén waves play a similar role.

In order to study further the cascades of kinetic and magnetic energy and helicity as well as cross-correlation, and the dynamical equilibria that can be reached between them, one needs large scale separation and large Reynolds number $R_{V}$. Models of MHD turbulence (see §4) may help unravel further these competing dynamics. Do we obtain $H_{m} \sim k^{-4}$ in the limit of high $R_{V}$ ? What role is played by the scales larger than the forcing (or the scale of the initial conditions)? Are there dual cascades in turbulent flows besides the one mentioned previously? (Note that it is already known that the magnetic energy is transferred both to large scale (following the magnetic helicity) and to small scales, through stretching by vorticity gradients; however, strictly speaking, $E_{m}$ is not an invariant but in the case when $E_{m} \gg E_{v}$, the total energy cascade is dominated by the magnetic energy.) Furthermore, what happens when the inverse cascade of magnetic helicity reaches the size of the vessel? Is there a rebound of $H_{m}$ and what effect would it have on the dynamics at smaller scales? Answers to these questions are not known. Note also that they are linked to the 2D Navier-Stokes case, underlying in fact the current debate around the interpretation of atmospheric data with a dual $\left(k^{-3}\right.$ at large scale and $k^{-5 / 3}$ at small scale, see, e.g., Gkioulekas \& Tung 2007 and references therein).

\section{Acknowledgements}

We dedicate this paper to Juri Toomre who has been an inspiration to several generations of researchers and who has conducted with his team magnificent computations of solar and stellar convection, leading the way to a deeper understanding of astrophysical flows. The computations reported here were performed, for the most part, at NCAR which is sponsored by the National Science Foundation.

\section{References}

Biskamp, D. \& Welter, H. 1989, Phys. Plasmas, B1, 1964

Politano, H., Pouquet, A. \& Sulem, P. L. 1989, Phys. Fluids B, 1, 2330

Mininni, P. D. \& Pouquet, A. 2009, Phys. Rev. E, 80, 025401(R)

Wang, X. G., Bhattacharjee, A. \& Ma, Z. W. 2000, J. Geophys. Res. - Space Physics, 105, 27633

Matthaeus, W. H. \& Lamkin, S. L. 1986, Phys. Fluids, 29, 2513

Servidio, S., Matthaeus, W. H., Shay, M. A., Dmitruk, P., Cassak, P. A. \& Wan, M. 2010, Phys. Plasmas, 17, 032315

Mininni, P. D. 2011, January issue, Ann. Rev. Fluid Mech., 43

Mininni, P. D., Alexakis, A. \& Pouquet, A. 2007, J. Plasma Phys.,73, 377

Sridhar, S. \& Goldreich, P. 1994, Astrophys. J., 432, 612

Montgomery, D. \& Matthaeus, W. H. 1995, Astrophys. J., 447, 706

Ng, C. S. \& Bhattacharjee, A. 1996, Astrophys. J., 465, 845

Iroshnikov, P. S. 1963, Sov. Astron., 7, 566

Kraichnan, R. H. 1965, Phys. Fluids, 8, 1385

Mininni, P., Montgomery, D. \& Pouquet, A. 2005, Phys. Fluids, 17, 035112

Dmitruk, P., Matthaeus, W. H. \& Gòmez, D. 2003, Phys. Plasmas, 10, 3584

Müller, W. C. \& Grappin, R. 2005, Phys. Rev. Lett., 95, 114502

Mason, J., Cattaneo, F. \& Boldyrev, S. 2008, Phys. Rev. E, 77, 036403

Brachet, M. E., Meiron, D. I., Orszag, S. A., Nickel, B. G., Morf, R. H. \& Frisch, U. 1983, J. Fluid Mech., 130, 411

Lee, E., Brachet, M. E., Pouquet, A., Mininni, P. \& Rosenberg, D. 2010, Phys. Rev.E, 81, 016318 
Goldreich, P. \& Shridar, S. 1995, Astrophys. J., 438, 763

Galtier, S., Pouquet, A. \& Mangeney, A. 2005, Phys. Plasmas, 12, 092310

Yurchyshyn, V., Yashiro, S., Abramenko,V., Wang, H. \& Gopalswamy, N. 2005, Astrophys. J., 619,599

Uritsky, V. M., Pudovkin, M I. \& Steen, A. 2001, J. Atmosph. Solar-Terrestrial Phys., 63, 1415

Müller, W. C. \& Biskamp, D. 2003, Phys. Rev. E, 67, 066302

Pouquet, A. 1993, Magnetohydrodynamic Turbulence, Les Houches Session XLVII, 139; Zahn, J.P. \& Zinn-Justin, J., Elsevier, eds.

Pouquet, A. 1996, Turbulence, Statistics and Structures: an Introduction, Springer-Verlag, Lecture Notes in Physics "Plasma Astrophysics" 468, 163, Chiuderi, C. \& Einaudi, G., eds.

Boldyrev, S. 2006 Phys. Rev. Lett., 96, 115002

Boldyrev, S. 2005, Astrophys. J. Letters, 626, L37

Politano, H. \& Pouquet, A. 1998, Geophys. Res. Lett., 25, 273

Mason, J., Cattaneo, F. \& Boldyrev, S. 2006, Phys. Rev. Lett., 97, 255002

Frisch, U., Pouquet, A., Léorat, J. \& Mazure, A. 1975, J. Fluid Mech., 68, 769

Pouquet, A., Frisch, U. \& Léorat, J. 1976 J. Fluid Mech., 77, 321

Müller, W. C. 2008, Private communication

Malapaka, S. K. 2009, PhD Thesis, "A study of magnetic helicity in decaying and forced 3DMHD turbulence," University of Bayreuth

Mininni, P. D. \& Pouquet, A. 2010, Phys. Fluids, 22, 035106

Pouquet, A. \& Patterson, G. S. 1978, J. Fluid Mech., 85, 305

Stribling, T. \& Matthaeus, W. H. 1990, Phys. Fluids, B2, 1979

Smith, C. W., Stawarz, J. E., Vasquez, B. J., Forman, M. A. \& MacBride, B. T. 2009, Phys. Rev. Lett., 103, 201101

Pouquet, A., Baerenzung, J., Pietarila Graham, J., Mininni, P. D., Politano, H. \& Ponty, Y. 2010, Notes on Numerical Fluid Mechanics, and Multidisciplinary Design, Springer Verlag, M. Deville, J.P. Sagaut \& T. Hiep eds., (see also arXiv:0904.4860)

Meneveau, C. \& Katz, J. 2000, Ann. Rev. Fluid Mech., 32, 1

Krstulovic, G., Mininni, P. D., Brachet, M. E. \& Pouquet, A. 2009, Phys. Rev. E, 79, 056304

Lu, E. T. \& Hamilton, R. J. 1991, Astrophys. J. Letters, 380, L89

Uritsky, V., Pouquet, A., Rosenberg, D. \& Mininni, P. D. 2010, "Structures in magnetohydrodynamics turbulence: detection and scaling," submitted to Phys. Rev. E, see arxiv:1007.0433

Uritsky, V. M., Paczuski, M., Davila, J. M. \& Jones, S. I. 2007, Phys. Rev. Lett., 99, 025001

Uritsky, V. M., Davila, J. M. E Jones, S. I. 2009, Phys. Rev. Lett., 103, 039502

Onsager, L. 1949, Supplemento al vol. VI, Serie IX del Nuovo-Cimento, 2, 279

Lee, T. D. 1952, Quart. Appl. Math, 10, 69

Kraichnan, R. H. 1975, J. Fluid Mech., 67, 155

Cichowlas, M., Bonaiti, P., Debbasc, F. E Brachet, M. E. 2005, Phys. Rev. Lett., 95, 264502

Krstulovic, G., Brachet, M. E. \& Pouquet, A. 2010, "Ideal two-dimensional dynamics of the Euler and MHD equations," in preparation

Okamoto, N., Yoshimatsu, K., Schneider, K., Farge, M. \& Kaneda, Y. 2007, Phys. Fluids, 19, 115109

Bandi, M. M., Chumakov, S. G. \& Connaughton, C. 2009, "On the probability distribution of power fluctuations in turbulence," submitted, see arXiv:0901.0743

Pietarila Graham, J., Holm, D., Mininni, P. D. \& Pouquet, A. 2010, "The effect of subfilter-scale physics on regularization models," to appear, Springer -Verlag, Conference on Quality and Reliability in Large Eddy Simulations ; see also arxiv.org/abs/1003.0335v1

Politano, H., Gomez, T. \& Pouquet, A. 2003, Phys. Rev. E, 68, 026315

Galtier, S. 2010, Phys. Rev. E, 77, 015302(R)

Pietarila Graham, J., Mininni, P. D. \& Pouquet, A. 2009, Phys. Rev. E, 80, 016313

Baerenzung, J., Politano, H.,. Ponty, Y. \& Pouquet, A. 2008, Phys. Rev. E, 78, 026310

Baerenzung, J., Mininni, P. D., Pouquet, A. \& Rosenberg, D. 2010, "Spectral Modeling of Turbulent Flows and the Role of Helicity in the presence of rotation," see also arXiv:0912.3414

Gkioulekas, E. \& Tung, K. K. 2007, Discrete Contin. Dyn. Syst. Ser. B, 7, 293 


\section{Discussion}

S. ToBIAs: This lack of universality in MHD turbulence is very interesting, but also very worrying in astrophysical/geophysical flows that often arises due to the presence of coherent structures but you find it in homogeneous isotropic turbulence. Is there any "phase information" you can pick out in your models to distinguish the initial conditions that behave so differently?

A. Pouquet: Well, there are subtleties. The MHD turbulence we generally study is isotropic insofar as we do not impose a mean field, but it is locally anisotropic at small scale. And the velocity and magnetic fields we use to force are large-scale ordered fields, for the velocity corresponding to a flow between two counter-rotating cylinders (the von Kàrman flow) and which can be viewed as modeling large-scale instabilities as can be found indeed in astrophysical flows. But to answer you about phase, no, I cannot pick up any information to explain why the magnetic energy grows in one case rather substantially (by a factor close to 6) and does not grow or decay as much (vis-à-vis its kinetic counterpart) in the other cases, except to say that one of the flows is "conducting" insofar as the current in the so-called impermeable domain (from which the whole flow is reconstructed when implementing the symmetries) is orthogonal to the walls, whereas in the other cases it is insulating, with the current in the walls of the impermeable domain. However, I might add that in that first case of strong domination of magnetic energy, at $t=0$, we have $\mathbf{b}=\omega$. Also note that, when observed, the excess of magnetic energy is clearly taking place in the gravest mode accessible to the system, and that when $E_{m} / E_{v}$ decays in the first flow, the energy spectrum shows sighs of being less steep. In other words, these classes of universality may well be not an intrinsic property of a given flow (initial condition) but may rather result from the internal complex dynamics of turbulent flows. Of course, this last result is obtained for late times at which the total energy and thus the Reynolds numbers have decreased measurably. In order to pursue such investigations, models such as those mentioned in $\S 4$, and the so-called shell models of turbulence as well (see e.g., Plunian \& Stepanov 2006) may prove quite useful.

A.S. BRun: Are you seeing evidence of the current sheet vortex in the high order structure functions?

A. Pouquet: Small-scale structures, such as current and vorticity sheets and rolls are the basis of the intermittency of turbulence, and as such are probably responsible for the variation with order of the anomalous exponents of structure functions, as shown e.g. in an analysis using wavelets (Okamoto et al. 2007). As I discussed in my talk, in solar active regions, there is observational evidence that the functional form of these exponents varies with the intensity of the flare (Yurchyshyn et al. 2005), and numerically as well it varies with the intensity of the imposed magnetic field (Müller \& Biskamp 2003). How different are the small-scale structures for an IK, K41 or WT spectrum? This is of course a good but difficult question, that perhaps can be addressed today using high-resolution direct numerical simulations (DNS). But what are we asking for precisely? Probably one could start by examining the structures associated with the extreme values of the velocity and magnetic field gradients.

A. Brandenburg: If $\tau_{A} / \tau_{N L}$ is a function of $\mathrm{k}$, you should expect kinetic and magnetic energy spectra to become non parallel. Can this be true also asymptotically? 

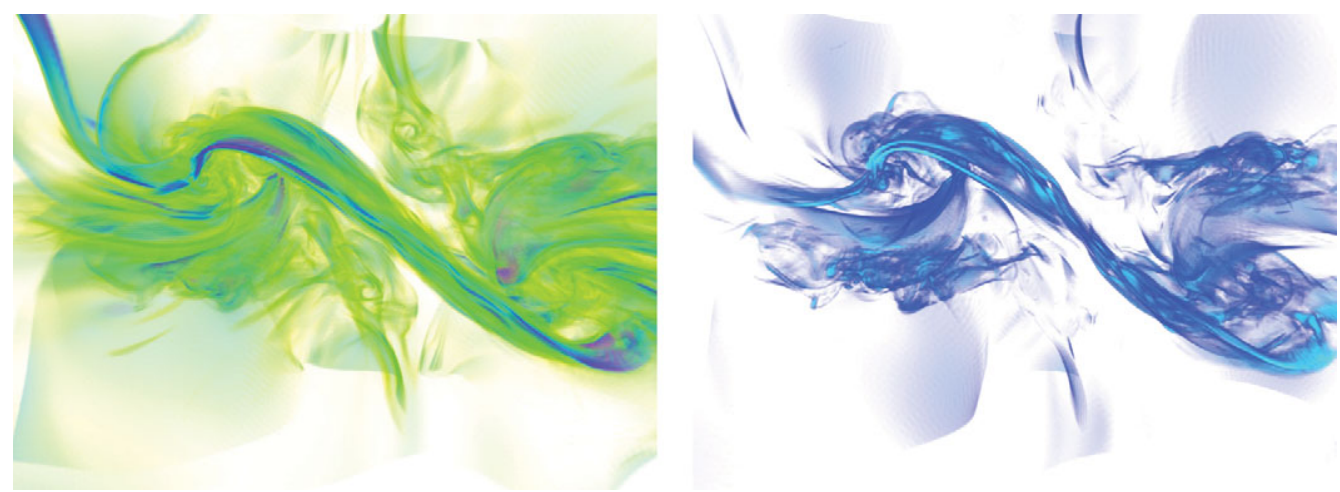

Figure 4. Snapshots of a zoom on the current density (left) [as in Fig. 3 but with a different perspective], and of the enstrophy density $\omega^{2}$ (right) at the same time $(t=4)$ as in Fig. 3 and for the same Taylor-Green (TG) flow with an Iroshnikov-Kraichnan spectrum (see Lee et al. 2010); $2048^{3}$ equivalent grid resolution with a code implementing the symmetries of the TG flow generalized to MHD (see $\S 2$ ). Note the overall spatial correlation between both sets of structures, but with more complexity in the vorticity field.

A. Pouquet: Yes indeed, there are measurable differences between the velocity and the magnetic filed inertial indices, as measured both in the solar wind (Podesta et al. 2007) and in highly resolved DNS (Mininni \& Pouquet 2010). Now, I suppose that you mean asymptotically in Reynolds number $R_{V}$ (let us assume for the sake of simplicity that the magnetic Prandtl number is equal to unity, which is the case for most of the numerical simulations I am describing here). But we have another parameter, which is time. It takes a time $\sim 1 / K_{0}$ to reach equipartition at wavenumber $K_{0}$, but as I increase $R_{V}$ by keeping the dissipation scale fixed, I have more and more wavenumbers available at large scale for which equipartition is not yet reached, leading to the possibility that the velocity and magnetic field energy spectra differ even at high Reynolds numbers. That does lead again to the question, though, of whether the breaking-down of universality we have observed in decaying flows occurs as well in the statistically steady case in the absence of an imposed uniform field.

H.K. Moffatt: The roll-up of current sheets suggests that these must coincide with vortex sheets which are still subject to Kelvin-Helmholtz instability (partially stabilized by the magnetic field). Can you explain the tendency for the formation of these currentvortex sheets?

A. Pouquet: Absolutely, vortex sheets roll-up as well, but the current and vorticity structures may not coincide exactly in space (see Fig. 4). Indeed, it is known that in two dimensions, at a neutral $\mathrm{X}$ point, the current is a dipole whereas the vorticity has a more complex structure (it is a quadrupole). In fact, when looking at the normalized correlation between $\mathbf{v}$ and $\mathbf{b}$, one observes that the large scales can be highly correlated (and/or anticorrelated, even if the total correlation integrated over the domain is weak) but that at the boundaries between large energy-containing regions or eddies, the structure of the correlation coefficient is quite complex (see, e.g, Meneguzzi et al. 1996). What these rolls are precisely has not been studied much. There is a possibility that they fit the description of so-called Alfvén vortices (see Kadomtsev \& Pogutse 1974), as observed in the solar wind by Alexandrova et al. (2006); but to my knowledge a Kelvin-Helmholtz instability of the development of these rolls (probably simpler when using the Elsässer fields since one can expect a more symmetric form in these variables) has not been performed yet. When 
doing so, one may want to take into consideration that we observe that the magnetic field lies within the current sheets which roll around its main direction. We have also observed at least in some cases that a clear rotational discontinuity develops (Lee et al. 2008), with two nearby sheets coming into close contact and with the magnetic field in each sheet in different directions (note that rotational discontinuities have been documented in the solar wind, see e.g. Whang 2004).

\section{References}

Plunian, F. \& Stepanov, R., 2006 J. Turbulence, 7, 39

Podesta, J. J., Roberts, D. A. \& Goldstein, M. L. 2007, Astrophys. J., 664, 543

Meneguzzi, M., Politano, H., Pouquet, A. \& Zolver, M. 1996, J. Comp. Phys., 123, 32

Kadomtsev, B. B. \& Pogutse, O. P. 1974, Z. Eksp. Teor. Fiz., 65, 575

Alexandrova, O., Mangeney, A., Maksimovic, M., Cornilleau-Wehrlin, N., Bosqued, J.-M. \& André, M. 2006, J. Geophys. Res., 111, A12208

Lee, E., Brachet, M., Pouquet, A., Mininni, P. \& Rosenberg, D. 2008, Phys. Rev.E, 78, 066401

Whang, Y. C. 2004, Nonlinear Processes Geophys., 11, 259 